\title{
Immunosuppressive effect of systemic administration of Lactobacillus rhamnosus KL37C-derived exopolysaccharide on the OVA-specific humoral response
}

\author{
MARTA CISZEK-LENDA ${ }^{\prime}$, BERNADETA NOWAK ${ }^{1}$, MAEGORZATA ŚRÓTTEK ${ }^{l}$, \\ SABINA GÓRSKA-FRĄCZEK ${ }^{2}$, ANDRZEJ GAMIAN², JANUSZ MARCINKIEWICZ \\ ${ }^{1}$ Department of Immunology, Jagiellonian University College of Medicine, Krakow, Poland \\ ${ }^{2}$ Institute of Immunology and Experimental Therapy, Polish Academy of Science, Wroclaw, Poland
}

\begin{abstract}
Lactobacilli, including Lactobacillus rhamnosus, commonly used probiotic bacteria, show the ability to produce biofilm. Whole lactic acid bacteria, as well as their cell-wall components show strain specific immunomodulatory properties. Exopolysaccharides (EPSS) are major components of extracellular polymeric substances and the lactobacilli biofilm matrix. However, in contrast to peptidoglycans or teichoic acids, the role of EPS in the modulation of the immune system by lactobacilli is still obscure. Previously, we have shown that crude EPS (cEPS) isolated from L. rhamnosus KL37C can effectively stimulate production of inflammatory mediators by macrophages in vitro. Moreover, EPS inhibits the production of anti-collagen specific immunoglobulin $G(\mathrm{IgG})$ in mice. Interestingly, the reduction of anti-collagen antibodies correlated with the amelioration of collagen-induced arthritis. However, the mechanism of EPS immunosuppressive activity remains unknown. In this paper we examine the ability of EPS to inhibit humoral response to ovalbumin (OVA). The experiments were performed in CBA mice, by immunizing animals with OVA in the presence of EPS or lipopolysaccharide (LPS). The results showed that EPS inhibited humoral response to OVA. Exopolysaccharide injected simultaneously with the antigen decreased the production of anti-OVA IgG, IgGI and IgG2a antibodies. Interestingly, EPS given together with LPS diminished its adjuvant properties by inhibiting OVA-specific IgG production. Therefore, our data indicate that EPS may exert opposing effects on antibody production in vivo to the adjuvant-like effect of whole lactic bacteria strains, as reported by others. Moreover, this study expands our understanding of a role of EPS in the "cross-talk" between biofilm forming bacteria and the immune system.
\end{abstract} biotics.

Key words: exopolysaccharides, humoral response, Lactobacillus rhamnosus, LPS, ovalbumin, pro-

(Centr Eur J Immunol 2012; 37 (4): 338-344)

\section{Introduction}

Exopolysaccharides (EPSs), major components of extracellular polymeric substances, are produced by bacteria during formation of biofilm [1]. However, in contrast to wellknown receptors of the bacteria cell-wall structures, such as Toll-like receptor 4 (TLR-4) for lipopolysaccharide (LPS) of Gram-negative bacteria or TLR-2 for peptydoglycans of
Gram-positive bacteria [2], it is not clear whether EPS can be recognized by the pathogen recognition receptors (PRRs). On the other hand, the immunoregulatory potential of EPS from various bacteria has been demonstrated in a number of papers [3-6]. Moreover, it is well established that EPS, a component of biofilm matrix, plays an important role in bacteria immune evasion and resistance to anti-bacterial agents, such as antibiotics and antiseptics [7-9].

Correspondence: Janusz Marcinkiewicz, Department of Immunology, Jagiellonian University Medical College, 18 Czysta Street, $31-121$ Kraków, Poland, e-mail: mmmarcin@cyf-kr.edu.pl 
It has been shown that EPSs from different Lactobacillus strains not only effectively alter the production of inflammatory mediators by the cells of innate immune system but can also affect antigen-specific humoral response [10, 11].

In our laboratory Lactobacillus rhamnosus KL37C, the high-level-EPS producer, have been selected out of several tested lactobacilli for investigations of EPS immunoregulatory properties. Recently, we have shown that crude EPS (cEPS) significantly ameliorates arthritis in the active model of collagen-induced arthritis (CIA) in mice, especially when LPS was used as adjuvant [11]. Independently, other reports have shown that oral administration of L. rhamnosus attenuates CIA and inhibits arthritogenic autoantibodies [12]. Therefore, it was reasonable to check whether systemic administration of cEPS affects the development of CIA and depresses the production of anti-collagen IgG. Indeed, cEPS strongly reduced the serum level of total collagen-specific IgG and of both measured subclasses of IgG, namely IgG1 and IgG2a [11]. The inhibitory effect of EPS on the humoral response was also confirmed by others [13].

The main aim of this study was to further evaluate the immunosuppressive potential of crude EPS derived from L. rhamnosus $\mathrm{KL} 37 \mathrm{C}$ in the OVA-model of antigen-specific antibody production in CBA mice. The effect of crude EPS was compared with that of lipopolysaccharide (LPS) and the selected polysaccharides derived from other sources. Finally, we compared the effect of crude EPS (extracellular polymeric substances) with that of purified form of EPS $\left(\mathrm{EPS}_{37}\right)$.

\section{Material and methods}

\section{Mice}

Inbred CBA/J mice (8-12 weeks of age, 18-22 g) were maintained in the Animal Breeding Unit, Department of Immunology, Jagiellonian University Medical College, Cracow. All mice were housed in the laboratory room with water and standard diet ad libitum. The authors were granted permission by the Local Ethical Committee to use mice in this study.

\section{EPS isolation}

Exopolysaccharides were obtained from L. rhamnosus KL37C strain isolated from the faeces of the human newborns and from Lactobacillus reuteri 133 from the gastrointestinal tract of Gai2-deficient mice which showed clinical symptoms of colitis $[14,15]$. Briefly, bacteria were stored at $-70^{\circ} \mathrm{C}$ in MRS broth supplemented with $10 \%$ glycerol. Bacteria were cultivated in supplemented MRS liquid broth (Oxoid, UK) under anaerobic conditions at $37^{\circ} \mathrm{C}$ for $48 \mathrm{~h}$. Cells were harvested by centrifugation at $8000 \mathrm{rpm}$ $\left(4^{\circ} \mathrm{C}, 30 \mathrm{~min}\right)$ and washed twice with phosphate buffer solution (PBS). Bacterial mass was suspended in water $(10 \mathrm{ml})$ and sonicated three times for $5 \mathrm{~min}$, in an ice bath. After centrifugation at $4000 \mathrm{rpm}\left(30 \mathrm{~min}, 4^{\circ} \mathrm{C}\right)$, the supernatant was centrifuged twice at $12000 \mathrm{rpm}, 4^{\circ} \mathrm{C}$ for $1 \mathrm{~h}$ and then precipitated with five volumes of cold ethanol $\left(-20^{\circ} \mathrm{C}\right.$, overnight). The precipitated material was recovered by centrifugation at $12000 \mathrm{rpm} 4^{\circ} \mathrm{C}$ for $20 \mathrm{~min}$ and freeze-dried. Isolation of EPSs was performed by gel filtration on the TSK HW-50 column $(1.6 \times 100 \mathrm{~cm})$ in $0.05 \mathrm{M}$ aqueous pyridine acetate buffer ( $\mathrm{pH}$ 5.6). The eluate was monitored with a Knauer differential refractometer. The first fraction, eluted in the void volume, contained polysaccharide (crude EPS) and was the subject of the present investigation.

\section{Purification of EPS $_{37}$}

Purification of crude EPS from L. rhamnosus KL37C was performed as described before [16]. The freeze-dried preparation of cEPS was dissolved in buffer (50 mM Tris$\mathrm{HCl} \mathrm{pH} \mathrm{7.5,} 10 \mathrm{mM} \mathrm{MgCl}_{2}$ ) and treated with DNase and RNase $\left(37^{\circ} \mathrm{C}, 6 \mathrm{~h}\right)$ with protease from Streptomyces griseus (both Sigma-Aldrich, Germany) $\left(37^{\circ} \mathrm{C}, 16 \mathrm{~h}\right)$, then dialyzed against water at $4^{\circ} \mathrm{C}$ for $24 \mathrm{~h}$. The resuspended polysaccharide was purified by ion-exchange chromatography on DEAE-Sephadex A-25, $1.6 \times 20 \mathrm{~cm}$, column (Pharmacia Fine Chemicals, Sweden). The pure EPS $_{37}$ was eluted with $20 \mathrm{mM}$ Tris buffer, $\mathrm{pH}$ 8.2. The fractions containing $\mathrm{EPS}_{37}$ were pooled, desalted by dialysis against water at $4{ }^{\circ} \mathrm{C}$ for $24 \mathrm{~h}$ and lyophilized.

\section{Mice immunization with OVA}

If not stated otherwise, mice were given intraperitoneally injection of OVA (200 mg/mouse) in the presence of LPS (Escherichia coli 011 : B4; $1 \mu \mathrm{g} /$ mouse; both Sigma-Aldrich, Germany), or/and with cEPS or EPS37 or EPS from $L$. reuteri 133 (cEPS133); $50 \mu \mathrm{g} /$ mouse twice, on day 0 and 14 . Seven days after the second immunization mice were bled and serum was collected. Level of OVA-specific antibodies was measured by ELISA.

\section{Measurement of serum OVA-specific antibody titer}

The level of antibodies specific to OVA in mouse serum was measured by ELISA test. Briefly, plates were coated overnight with OVA $(5 \mu \mathrm{g} / \mathrm{ml})$. Serial dilutions of mouse serum in PBS were applied to antigen-coated wells for $1 \mathrm{~h}$ at room temperature. Immunoglobulins in tested serum samples were detected using biotin-conjugated antibodies against IgG (Sigma-Aldrich, Germany), IgG1 (MP Biomedicals, USA), or IgG2a (Southern Biotech, USA). The amount of antibody was expressed in arbitrary ELISA units calculated from anti-OVA titer: 1 Unit $=1 / 100$ titers of antibodies specific to OVA.

\section{Statistical analysis}

Statistical significance of differences between groups was analyzed using one-way ANOVA, followed, if significant, by a Tukey test for post hoc comparison. Results are expressed as mean \pm SEM values. A $p$-value $<0.05$ was con- 
A

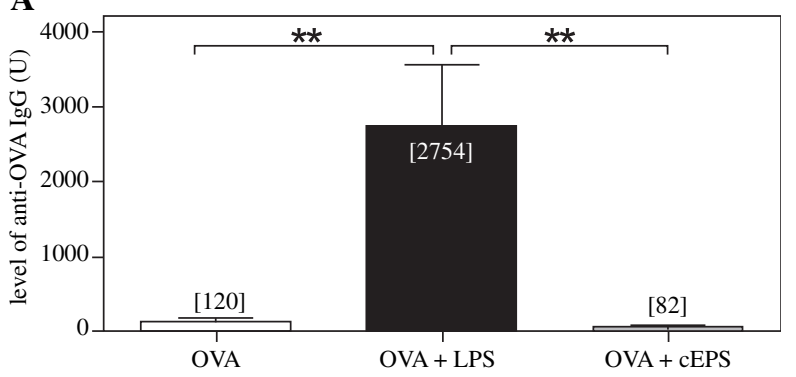

C

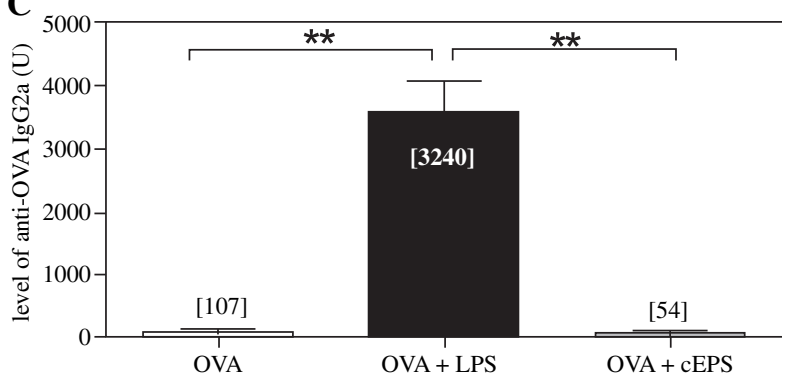

sidered statistically significant. Analysis was performed using Graphpad Prism v. 5.01 (GraphPad Software, Inc., USA).

\section{Results}

\section{Effects of crude EPS on the level of OVA-specific IgG}

To examine the immunosuppressive potential of crude EPS on humoral response in mice we used the protocol of OVA-specific antibody production and the effect of cEPS was compared with that of LPS. In comparison to the preliminary studies we extended our present investigation on a measurement of IgG subclasses [11]. Lipopolysaccharide, a well-known bacterial stimulator of B cell proliferation [17] behaved as a strong adjuvant in all our experiments, enhancing significantly OVA-specific IgG of all tested subclasses (10-20-fold enhancement). Crude EPS, in contrast to LPS, slightly inhibited the serum concentration of OVA-specific IgG (total IgG, IgG1 and IgG2a), as shown in Fig. 1. We did not find any effect of cEPS on anti-OVA IgM production in our experimental set-up (data not shown). On the other hand, cEPS not only reduced the production of OVA-specific antibodies when injected with OVA alone, but also abolished the adjuvant effect of LPS. Indeed, when cEPS was given simultaneously with antigen and LPS, the serum concentration of anti-OVA IgG was markedly reduced (Fig. 2).

\section{Different routes of EPS injection and the production of OVA-specific IgG}

To exclude the effect of local interactions between crude EPS and OVA, the antigen and cEPS were administered by different routes. The results shown in Fig. 3 clearly indicate

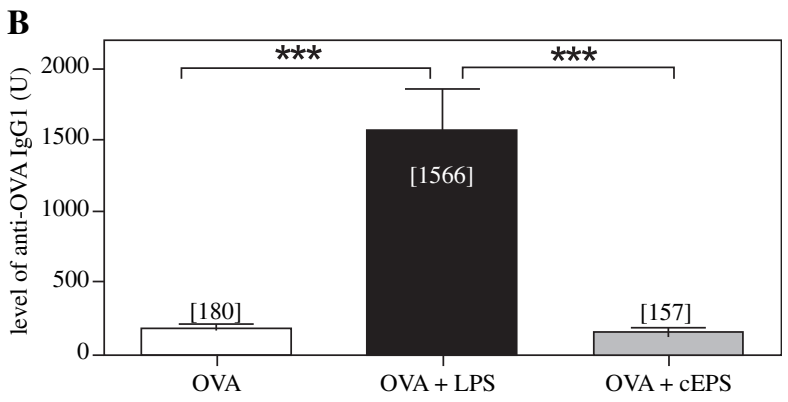

Fig. 1. The effect of crude EPS on OVA-specific IgG, IgG1 and IgG2a production. Mice were immunized intraperitoneally with $200 \mu \mathrm{g}$ OVA alone, OVA together with LPS $(1 \mu \mathrm{g} /$ mouse $)$ or OVA with cEPS $(50 \mu \mathrm{g} / \mathrm{mouse})$ on day 0 and 14 . The level of IgG (A), IgG1 (B) and IgG2a (C) specific for OVA were measured by ELISA 7 days after boost immunization. The results are expressed in arbitrary units: $1 \mathrm{U}=1 / 100$ titer of $\mathrm{IgG}$ anti-OVA \pm $\operatorname{SEM}(n=10)$. Results are mean of two independent experiments. The levels of IgGs are shown in brackets. Statistical significance between levels of IgG are marked as: ${ }^{* *} p<0.01 ;{ }^{* * *} p<0.001$

that cEPS injected intraperitoneally (i.p.) or intravenously (i.v.) leads to reduction of the production of anti-OVA IgG. However, the strongest suppression was observed after intravenous administration of cEPS. By contrast, subcutaneous injection (s.c.) of cEPS only slightly increased the serum level of OVA-specific IgG. In all experiments OVA was applied intraperitoneally.

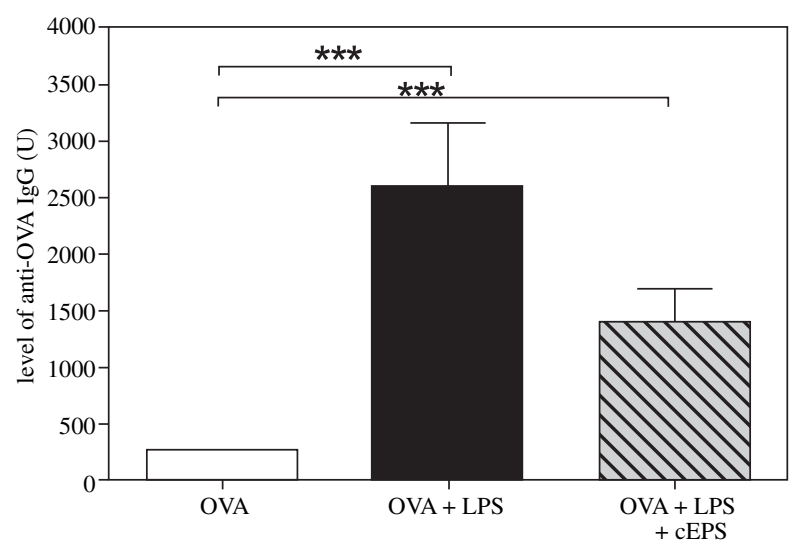

Fig. 2. Anti-adjuvant effect of cEPS. Mice were immunized intraperitoneally with $200 \mu \mathrm{g}$ OVA alone, OVA together with LPS $(1 \mu \mathrm{g} / \mathrm{mouse})$ or OVA together with LPS and cEPS ( $50 \mu \mathrm{g} /$ mouse) on day 0 and 14 . The level of OVA-specific IgG was measured by ELISA in individual sera collected 7 days after boost immunization. The results are expressed in arbitrary units representing $1 \mathrm{U}=1 / 100$ titer of $\operatorname{IgG}$ anti-OVA $\pm \operatorname{SEM}(n=15)$. Results represent three independent experiments. Statistical significance between levels of IgG are marked as: ${ }^{* * *} p<0.001$. 


\section{The ability of EPS to inhibit OVA-specific humoral response is not restricted to cEPS derived from human L. rhamnosus KL37C}

Previously we have shown that the ability of cEPS from L. rhamnosus KL37C to stimulate the production of cytokines by macrophages differs from that of EPSs derived from other Lactobacillus strains [10]. To examine whether EPS-dependent suppression of humoral response is also strain-specific we compared the effect of cEPS from L. rhamnosus KL37C with that of other crude EPS isolated from mice L. reuteri 133 and human L. rhamnosus GG. As shown in Fig. 4, the production of total IgG anti-OVA was markedly inhibited by $\mathrm{cEPS}_{133}$. The similar suppressor effect was achieved when we used EPS from L. rhamnosus GG (data not shown). Nevertheless, the strongest reduction of serum OVA-specific IgG was caused by cEPS from L. rhamnosus KL37C.

\section{Effects of crude and purified EPSs on the OVA- specific IgG production}

Finally, we compared the suppressor potential of purified EPS $\left(\mathrm{EPS}_{37}\right)$ with that of the cEPS to examine whether the polysaccharide, but not the other components of crude EPS is responsible for the reduction of OVA-specific IgG production. Administration of EPS $_{37}$ together with OVA during the primary and secondary immunization resulted in the inhibition of antigen-specific humoral response to a similar extent to that observed after administration of cEPS. Moreover, EPS $_{37}$ showed also anti-adjuvant effect by neutralization of the LPS-dependent enhancement of serum anti-OVA IgG (Fig. 5). Thus, these data suggest that the contribution of other components of extracellular polymeric substances, such as proteins, nucleic acids, are negligible in the observed suppression of OVA-specific humoral response.

\section{Discussion}

Lactic acid bacteria, the major probiotic bacteria, show strain-specific intrinsic immunoregulatory properties as was reported in a number of papers $[18,19]$. Wide spectrum of lactobacilli immunoregulatory activities includes a modulation of effector and regulatory $\mathrm{T}$ cell subsets, interactions with dendritic-cells, macrophages and B cells, with the mucosal tissue-associated cells especially [19-23]. Indeed, it has been demonstrated that the oral administration of some lactobacilli strains can induce oral tolerance. Namely, total and the antigen-specific IgE production was inhibited in atopic dermatitis model NC/Nga mice [24]. On the other hand, some lactobacilli strains were used as adjuvants to enhance the production of IgA in the MALT system [25, 26]. Moreover, lactobacilli have been shown to increase the immunogenecity of orally administered vaccines (e.g. rotavirus, cholera vaccines) $[27,28]$. Such distinct and even

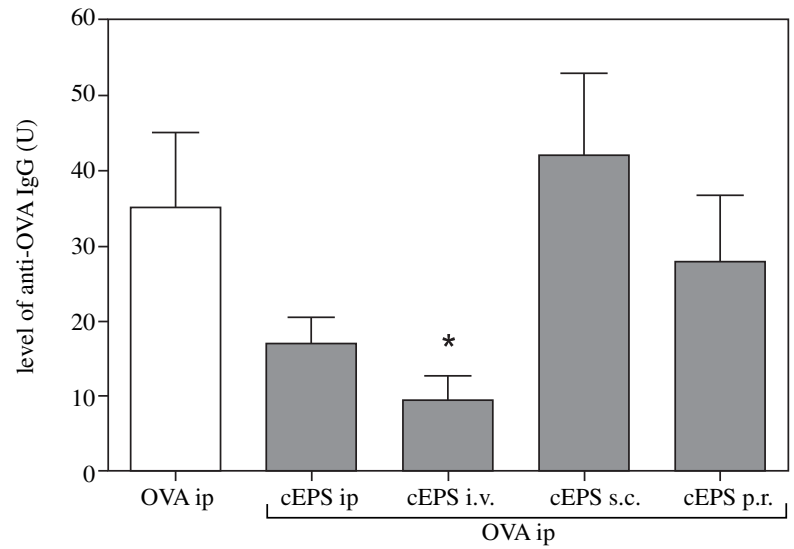

Fig. 3. Effects of crude EPS on the level of OVA-specific IgG alternative routes of cEPS injection. Mice were immunized with $200 \mu \mathrm{g}$ OVA alone or OVA together with cEPS $(50 \mu \mathrm{g} / \mathrm{mouse})$ on day 0 and 14. cEPS was administered intraperitoneally (i.p.), intravenously (i.v.), subcutaneously (s.c.) or rectally (p.r.). In all cases OVA was administered intraperitoneally. The level of OVAspecific IgG in serum from individual mice was measured by ELISA 7 days after boost immunization. The results are expressed in arbitrary units representing $1 \mathrm{U}=1 / 100$ titer of IgG anti-OVA $\pm \operatorname{SEM}(n=5)$. The results show one representative experiment. ${ }^{*} p<0.05$ OVA vs. OVA + cEPS i.v.

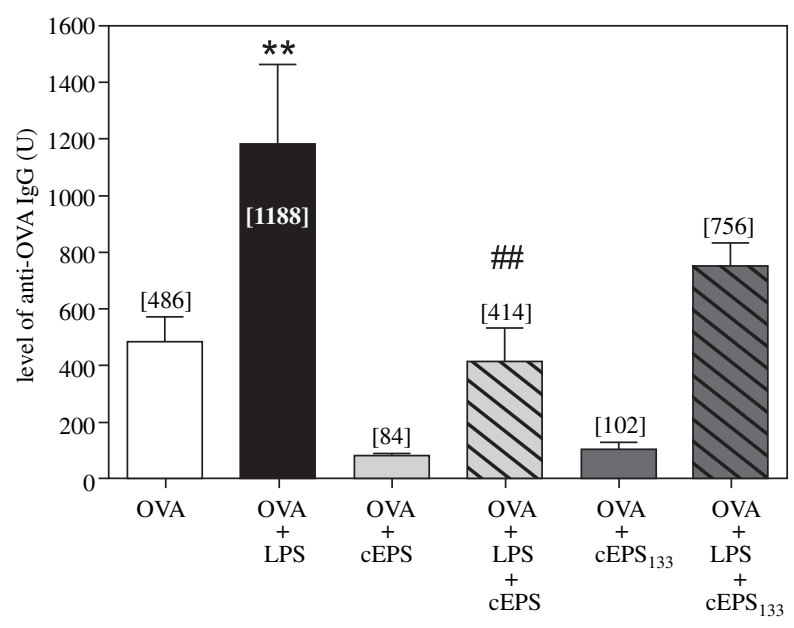

Fig. 4. The ability to inhibit OVA-specific humoral response is not restricted to cEPS from L. rhamnosus KL37C. Mice were immunized intraperitoneally with $200 \mu \mathrm{g}$ OVA alone, OVA together with LPS $(1 \mu \mathrm{g} /$ mouse) or OVA with cEPS (50 $\mu \mathrm{g} /$ mouse) or OVA with cEPS133 (50 $\mu \mathrm{g} /$ mouse) on day 0 and 14. The level of IgG specific for OVA were measured by ELISA 7 days after boost immunization. The results are expressed in arbitrary units representing $1 \mathrm{U}=1 / 100$ titer of $\mathrm{IgG}$ anti-OVA $\pm \operatorname{SEM}(n=5)$. The results show one representative experiment. The levels of $\mathrm{IgG}$ are shown in brackets ${ }^{* *} p<0.01$ OVA vs. LPS; ${ }^{\# \#} p<0.01$ OVA + LPS vs. OVA + LPS + cEPS 

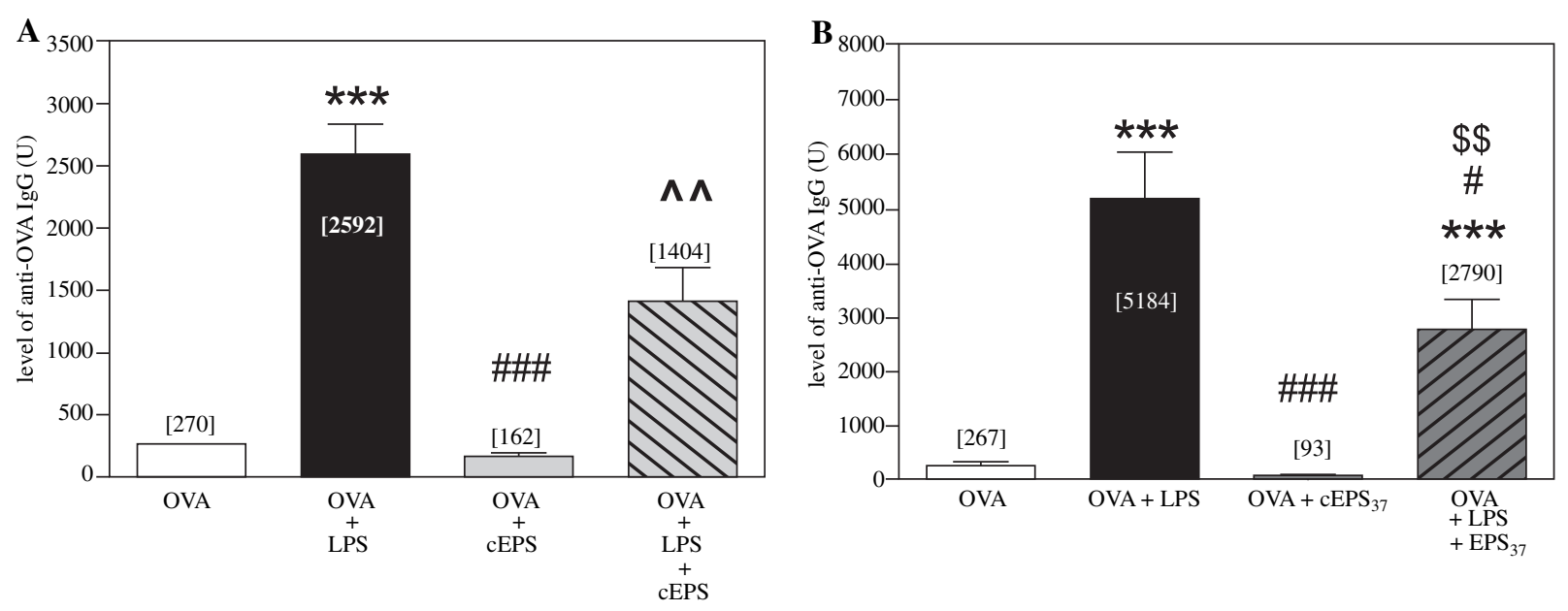

Fig. 5. Comparison of the effect of crude EPS (A) and purified $\mathrm{EPS}_{37}$ (B) on OVA-specific antibody production. Mice were immunized intraperitoneally with $200 \mu \mathrm{g}$ OVA alone, OVA together with LPS ( $1 \mu \mathrm{g} / \mathrm{mouse}$ ) or OVA with cEPS (50 $\mu \mathrm{g} / \mathrm{mouse})$ or OVA with $\mathrm{EPS}_{37}(50 \mu \mathrm{g} / \mathrm{mouse})$ on day 0 and 14. The level of IgG specific for OVA were measured by ELISA 7 days after boost immunization. The results are expressed in arbitrary units representing $1 \mathrm{U}=1 / 100$ titer of $\operatorname{IgG}$ anti-OVA $\pm \operatorname{SEM}(n=10)$. Results represent the mean of two independent experiments. The levels of IgG are shown in brackets. ${ }^{* * *} p<0.001$ vs. OVA; ${ }^{\#} p<0.05$ vs. LPS; ${ }^{\# \#} p<0.001$ vs. LPS; ${ }^{\wedge} p<<0.01$ vs. cEPS; $\$ \$ p<0.01$ vs. EPS $_{37}$

opposite immunomodulatory effects (enhancement vs. suppression of humoral response) of various lactobacilli may be explained by an extreme structural diversity of the cell wall components, especially due to the presence of different sugar monomers [29, 30]. Interestingly, it has been documented that EPSs, the major components of lactobacilli biofilm matrix, show a significant impact on lactic bacteria immunoregulatory properties, including enhancement of the humoral response [3]. Moreover, to our knowledge, apart from our investigations [11], only a few reports have described the immunosuppressive effects of bacterial EPSs $[5,31]$. All these data support a common opinion that biological functions of lactobacilli (EPSs) are not only strainspecific but the final effect also depends on a route of bacteria (EPS) administration, i.e. versus oral administration [32]. However, detailed mechanism of action of EPSs remains unclear.

In the present study we examined the suppressor activity of crude EPS derived from L. rhamnosus KL37C in the OVA-model of antigen-specific antibody production in CBA mice. In our basal experimental system mice were injected intraperitoneally with the antigen (OVA), adjuvant (LPS) and cEPS. Therefore, in contrast to other experimental models, restricted to oral administration of both EPS and antigen $[33,34]$ we could observe the effects of EPS on the systemic immune system. Importantly, we used the same route of OVA immunization, as the one we used previously for collagen to induce the CIA. Our present data indicate that cEPS inhibits the production of OVA-specific IgG in a similar way as it suppressed the production of anti-collagen $\mathrm{IgG}$ in the CIA [11]. In both unrelated antigenic systems the strongest reduction of serum level of antigen-specific antibodies was observed in IgG2a subclasses. It suggests that a polarization of $\mathrm{T}$ helper subsets occurs under the influence of cEPS. Moreover, the present study confirmed "antiadjuvant" properties of cEPS as it was able to neutralize the LPS-dependent enhancement of OVA-specific IgG production.

Nevertheless, it is still not known how cEPS attenuates the activity of LPS and reduces the serum level of antigenspecific IgG. We can speculate that at least two independent mechanisms may be responsible for the cEPS-dependent suppression of OVA-specific IgG production. Firstly, cEPS may interact directly with LPS reducing its contact with the antigen presenting cells. Secondly, cEPS may induce $\mathrm{T}$ regulatory cells (e.g. T suppressor cells in the spleen) and indirectly inhibit LPS-dependent proliferation of B cells. However, to confirm the above hypothesis we need stronger experimental data.

Importantly, in the present study we have shown for the first time that purified $\mathrm{EPS}_{37}$ exerted the same inhibitory properties as the crude EPS. It is of importance for further investigations as it would be easier to determinate receptor(s) specific for extracellular polysaccharides, especially for those which are components of a biofilm matrix, using a purified EPS.

In conclusion, further studies are necessary to confirm the hypothesis on the mechanism of cEPS-dependent suppression, to decide whether biofilm components such as EPS interact with PRRs of the cells of innate immune system and whether EPS may be used as immunomodulator with defined properties. Finally, in our opinion, a major gap 
in our knowledge about mechanisms of immunomodulation by probiotics (including biofilm EPS) concerns their diversity and their fate in vivo. Therefore, in the future, immunoregulatory functions of bacterial (probiotic) polysaccharides of a well-characterized structure should be examined in vitro and then confirmed in animal experimental models.

This study was supported by the grant from the National Science Centre No. K/PBW/000559.

\section{References}

1. Vu B, Chen M, Crawford RJ, Ivanova EP (2009): Bacterial extracellular polysaccharides involved in biofilm formation. Molecules 14: 2535-2554.

2. Takeuchi O, Hoshino K, Kawai T, et al. (1999): Differential roles of TLR2 and TLR4 in recognition of gram-negative and gram-positive bacterial cell wall components. Immunity 11: 443-451.

3. Vinderola G, Perdigón G, Duarte J, et al. (2006): Effects of the oral administration of the exopolysaccharide produced by Lactobacillus kefiranofaciens on the gut mucosal immunity. Cytokine 36: 254-260.

4. Chabot S, Yu H-Lig, De Léséleu L (2001): Exopolysaccharides from Lactobacillus rhamnosus RW-9595M stimulate TNF, IL-6 and IL-12 in human and mouse cultured immunocompetent cells and IFN- $\gamma$ in mouse splenocytes. Lait 81: 683-697.

5. Bleau C, Monges A, Rashidan K, et al. (2010): Intermediate chains of exopolysaccharides from Lactobacillus rhamnosus RW-9595M increase IL-10 production by macrophages. J Appl Microbiol 108: 666-675.

6. Wu MH, Pan TM, Wu YJ, et al. (2010): Exopolysaccharide activities from probiotic bifidobacterium: immunomodulatory effects (on J774A.1 macrophages) and antimicrobial properties. Int J Food Microbiol 144: 104-110.

7. Ruas-Madiedo P, Gueimonde M, Margolles A, et al. (2006): Exopolysaccharides produced by probiotic strains modify the adhesion of probiotics and enteropathogens to human intestinal mucus. J Food Prot 69: 2011-2015.

8. Al-Mathkhury HJ, Ali AS, Ghafil JA (2011): Antagonistic effect of bacteriocin against urinary catheter associated Pseudomonas aeruginosa biofilm. N Am J Med Sci 3: 367370.

9. Høiby N, Bjarnsholt T, Givskov M, et al. (2010): Antibiotic resistance of bacterial biofilms. Int J Antimicrob Agents 35: 322-332.

10. Ciszek-Lenda M, Strus M, Górska-Frączek S, et al. (2011): Strain specific immunostimulatory potential of lactobacilliderived exopolysaccharides. Centr Eur J Immunol 36: 121-129.

11. Nowak B, Ciszek-Lenda M, Śróttek M, et al. (2012): Lactobacillus rhamnosus exopolysaccharide ameliorates arthritis induced by the systemic injection of collagen and lipopolysaccharide in DBA/1 mice. Arch Immunol Ther Exp (Warsz) 60: 211-220.

12. Baharav E, Mor F, Halpern M, Weinberger A (2004): Lactobacillus $G G$ bacteria ameliorate arthritis in Lewis rats. J Nutr 134: 1964-1969.

13. Sugihara R, Yoshimura M, Mori M, et al. (2000): Prevention of collagen-induced arthritis in DBA/1 mice by oral adminis- tration of AZ-9, a bacterial polysaccharide from Klebsiella oxytoca. Immunopharmacology 49: 325-333.

14. Lipiński T, Jones C, Lemercinier X, et al. (2003): Structural analysis of the Lactobacillus rhamnosus strain KL37C exopolysaccharide. Carbohydr Res 338: 605-609.

15. Rudolph U, Finegold MJ, Rich SS, et al. (1995): Ulcerative colitis and adenocarcinoma of the colon in $\mathrm{G}$ alpha i2-deficient mice. Nat Genet 10: 143-150.

16. Górska-Frączek S, Sandström C, Kenne L, et al. (2011): Structural studies of the exopolysaccharide consisting of a nonasaccharide repeating unit isolated from Lactobacillus rhamnosus KL37B. Carbohydr Res 346: 2926-2932.

17. Cavallo T, Granholm NA (1990): Lipopolysaccharide from gram-negative bacteria enhances polyclonal B cell activation and exacerbates nephritis in MRL/lpr mice. Clin Exp Immunol 82: 515-521.

18. Wells JM, Rossi O, Meijerink M, van Baarlen P (2011): Epithelial crosstalk at the microbiota-mucosal interface. Proc Natl Acad Sci U S A 108 Suppl 1: 4607-4614.

19. Marcinkiewicz J, Ciszek M, Bobek M, et al. (2007): Differential inflammatory mediator response in vitro from murine macrophages to lactobacilli and pathogenic intestinal bacteria. Int J Exp Pathol 88: 155-164.

20. Jeon SG, Kayama H, Ueda Y, et al. (2012): Probiotic Bifidobacterium breve induces IL-10-producing $\operatorname{Tr} 1$ cells in the colon. PLoS Pathog 8: e1002714.

21. Christensen HR, Frøkiaer H, Pestka JJ (2002): Lactobacilli differentially modulate expression of cytokines and maturation surface markers in murine dendritic cells. J Immunol 168: 171-178.

22. Kitazawa H, Yamaguchi T, Fujimoto Y, Itoh T (1993): Comparative activity of B-cell mitogen, a phosphopolysaccharide produced by Lactococcus lactis ssp. cremoris on various lymphocytes. Anim Sci Technol 64: 604-607.

23. Bernardo D, Sánchez B, Al-Hassi HO, et al. (2012): Microbiota/host crosstalk biomarkers: regulatory response of human intestinal dendritic cells exposed to Lactobacillus extracellular encrypted peptide. PLoS One 7: e36262.

24. Segawa S, Hayashi A, Nakakita Y, et al. (2008): Oral administration of heat-killed Lactobacillus brevis SBC8803 ameliorates the development of dermatitis and inhibits immunoglobulin $\mathrm{E}$ production in atopic dermatitis model NC/Nga mice. Biol Pharm Bull 31: 884-889.

25. Van Overtvelt L, Moussu H, Horiot S, et al. (2010): Lactic acid bacteria as adjuvants for sublingual allergy vaccines. Vaccine 28: 2986-2992.

26. Malin M, Suomalainen H, Saxelin M, Isolauri E (1996): Promotion of IgA immune response in patients with Crohn's disease by oral bacteriotherapy with Lactobacillus GG. Ann Nutr Metab 40: 137-145.

27. Zhang W, Azevedo MS, Wen K, et al. Probiotic Lactobacillus acidophilus enhances the immunogenicity of an oral rotavirus vaccine in gnotobiotic pigs. Vaccine 26: 3655-3661.

28. Paineau D, Carcano D, Leyer G, et al. Effects of seven potential probiotic strains on specific immune responses in healthy adults: a double-blind, randomized, controlled trial. FEMS Immunol Med Microbiol 53: 107-113.

29. De Vuyst L, Degeest B (1999): Heteropolysaccharides from lactic acid bacteria. FEMS Microbiol Rev 23: 153-177.

30. Górska S, Grycko P, Rybka J, Gamian A (2007): Exopolysaccharides of lactic acid bacteria: structure and biosynthesis. Postepy Hig Med Dosw 61: 805-818.

31. Sugihara R, Oiso Y, Matsumoto Y, Ohmori H (2001): Production of an immunosuppressive polysaccharide, AZ9, in the 
culture of Klebsiella oxytoca strain TNM3. J Biosci Bioeng 92: 485-487.

32. Finamore A, Roselli M, Britti MS, et al. (2012): Lactobacillus rhamnosus GG and Bifidobacterium animalis MB5 induce intestinal but not systemic antigen-specific hyporesponsiveness in ovalbumin-immunized rats. J Nutr 142: 375-381.

33. Sengül N, Işık S, Aslım B, et al. (2011): The effect of exopolysaccharide-producing probiotic strains on gut oxidative damage in experimental colitis. Dig Dis Sci 56: 707-714.

34. Huibregtse IL, Snoeck V, de Creus A, et al. (2007): Induction of ovalbumin-specific tolerance by oral administration of $\mathrm{Lac}$ tococcus lactis secreting ovalbumin. Gastroenterology 133: 517-528. 